\title{
The Elimination of Abuse and Dependence in DSM-5 Substance Use Disorders: What Does This Mean for Treatment?
}

\author{
Albert M. Kopak • Steven L. Proctor • \\ Norman G. Hoffmann
}

Published online: 13 May 2014

(C) Springer International Publishing AG 2014

\begin{abstract}
The recently released Diagnostic and Statistical Manual of Mental Disorders, fifth edition (DSM-5) marks the beginning of a new chapter in the diagnosis and treatment of substance use disorders. This article provides a brief overview of the changes to the criteria and how they will be used to formulate the new diagnostic index. Recent research, although limited given the timeliness of the revision, has demonstrated consistent findings with regard to the compatibility between DSM-IV and DSM-5 criteria, predominantly related to the most serious cases of substance use. There is much more variation in how milder cases will be addressed with the DSM-5 relative to the prior version. These diagnoses have significant implications for treatment, but it is imperative to acknowledge prognoses are based on the assumption that DSM-5 substance use disorders are qualitatively similar but range in severity from mild to severe. This may be a controversial approach, given that some preliminary research and lively discussion suggests the new diagnostic scheme may encompass qualitatively unique disorders ranked on a continuum. Treatment goals must take these underlying issues into account if substance use disorders are to be addressed successfully. The DSM-5 represents advancement in the diagnosis of substance use disorders, but many questions related to
\end{abstract}

\footnotetext{
A. M. Kopak ( $\square)$

Department of Criminology \& Criminal Justice, Western Carolina University, 413-A Belk, Cullowhee, NC 28723, USA

e-mail: amkopak@wcu.edu

S. L. Proctor

Department of Psychology, Louisiana State University, 236 Audubon

Hall, Baton Rouge, LA 70803, USA

e-mail: sproct2@tigers.1su.edu

N. G. Hoffmann

Department of Psychology, Western Carolina University, 301 Killian, Cullowhee, NC 28723, USA

e-mail: evinceassessment@aol.com
}

how it will inform treatment remain unanswered. Implications for future research are also discussed in this review.

Keywords DSM-5 - Substance use disorders · Diagnostic criteria $\cdot$ Alcohol $\cdot$ Illicit drugs $\cdot$ DSM-IV

\section{Introduction}

The fifth edition of the Diagnostic and Statistical Manual of Mental Disorders (DSM-5) [1] was released in May 2013 with substantive changes made to the DSM-IV diagnostic criteria for substance use disorders (SUDs). The most notable change was the collapse of the abuse and dependence designations into a single severity index. The abuse and dependence classifications of the DSM-IV were intended to be related yet distinct clinical syndromes. Abuse was defined as a maladaptive pattern of use leading to clinically significant impairment or distress over a 12-month period [2]. Dependence was similarly defined as continued substance use despite behavioral impairment or distress in the same 12-month period, but there was a marked difference in the type of indicators used to diagnose dependence relative to abuse.

An abuse diagnosis was recognized through the endorsement of at least one of four relevant criteria. These four indicators included failure to fulfill major role obligations at home, school, or work; continued substance use in hazardous situations (e.g., driving an automobile while impaired); recurrent legal problems related to substance use; and persistent use despite recurrent social or interpersonal problems attributed to use. All of these criteria were included in the DSM-5, with the exception of the recurrent substance-related legal problems criterion. The DSM-5 also introduced an additional criterion representing craving or a strong desire to use a substance.

A dependence diagnosis was based on the endorsement of a minimum of three of seven clearly defined 
criteria. These seven indicators included the development of tolerance to a substance, withdrawal symptoms, unplanned use, desire and/or inability to reduce or stop use, a great deal of time related to substance use (e.g., obtaining, using, or recovering from the effects of a substance), continued use despite the development or exacerbation of medical or psychological problems, and forfeiture of recreational or other activities because of substance use. All of these seven criteria were retained in the DSM-5.

The DSM-5 is based on an 11-item severity index for SUD diagnoses, but several iterations have dealt with the thresholds within the index. The initial formulation included 'moderate' and 'severe' designations only, with those who endorsed two or three criteria falling into the moderate category and those who endorsed four or more criteria placed in the severe group. This scheme was revised, however, based largely on evidence demonstrative of a lenient threshold which tended to include those with low levels of substance involvement [3]. In other words, the two-criterion threshold for a moderate SUD had the potential to diagnose low-level or mild cases which were unlikely to have multiple indicators of compulsive or problematic substance use. Likewise, use of a four-criterion threshold for 'severe' cases meant the most serious diagnosis was reached on a relatively low point in the continuum (i.e., less than half the total number of criteria).

In response, the symptom thresholds were raised and an additional SUD category was created to reflect less severe or 'mild' cases. Thus, the DSM-5 currently includes three SUD designations: 'mild,' 'moderate,' and 'severe.' This means those who endorse two or three criteria will be assigned a mild SUD, those with four or five positive criteria will be assigned a moderate SUD, and those with six or more positive criteria will be diagnosed with a severe SUD [1].

The DSM has been at the heart of many debates since its inception, and this trend continues with the fifth edition. Researchers and American Psychological Association (APA) Substance Use Work Group committee members are deeply interested in the reliability and validity of the 11 criteria to accurately diagnose problematic patterns of substance use, while clinicians require precise diagnostic instruments to serve as the basis for effective treatment plans. Debates regarding the nature of criteria take place not only within these groups, but also between them. One major goal of the revision was to address some of the limitations in the prior diagnostic scheme by eliminating separate sets of criteria for abuse and dependence to make them easier for clinicians to use [4]. This, like many other objectives for the DSM-5, will need to be studied further to determine whether or not they are met.

\section{Recent Literature Devoted to the DSM-5 Criteria}

Given the recency of the finalized revision and its release, there is a relative paucity of research focused on the implications of the new diagnostic criteria. The vast majority of existing studies have concentrated on how the abuse and dependence designations of the DSM-IV compare to the index of the DSM-5. One focal issue which has arisen as a result of the collapse of the two distinct DSM-IV diagnoses (i.e., abuse and dependence) is the increase in the prevalence of SUDs. Evidence has shown this increase in diagnosable cases can range anywhere from $<1-11 \%$, due primarily to the occurrence of 'diagnostic orphans' (i.e., those endorsing one to two DSM-IV dependence criteria, yet no abuse criteria) now receiving a SUD diagnosis under the DSM-5 $[5,6,7 \cdot 8-11,12 \cdot]$.

Evidence also shows certain patterns in the convergence of the two approaches $[7 \bullet, 12 \bullet]$. For instance, the majority of cases which met DSM-IV abuse criteria will not receive a DSM-5 diagnosis because they fail to meet the minimum two-criterion threshold for a mild SUD. Most DSM-IV abuse cases, which also endorsed two dependence criteria, will now receive a mild SUD designation, but some who endorsed multiple (i.e., two or three) abuse criteria and two to three dependence criteria will now be classified with a moderate SUD. DSM-IV dependence cases who met three dependence criteria and no more than two abuse criteria will be classified as DSM-5 moderate SUDs, while almost all of those who met four or more dependence criteria will be classified as severe SUDs.

Based on the cursory information currently available, there are some strengths of the DSM-5 classification scheme relative to the abuse and dependence designations in the DSM-IV. Research with alcohol use has demonstrated the utility of examining this behavior and related problems along a continuum rather than as a comparison of latent classes representative of abuse and dependence [13]. Based on this evidence and findings from recent comparisons between the DSM-IV and DSM-5 criteria $[5,6,10]$, combining abuse and dependence into a single dimension seems to be a logical approach to better capture the severity of SUDs. This can be clearly exhibited in a hypothetical case which displayed a combination of abuse and dependence (and the corresponding minimum four criteria) and will now be addressed as a more severe disorder compared with distinct abuse and dependence cases under the DSM-IV. This is largely due to the fact that the number of positive criteria once required to achieve abuse and dependence diagnoses will now fall into the moderate DSM-5 SUD classification, at minimum. Despite some researchers' adherence to the conceptual distinctions between abuse and dependence [14], the new scheme seems to be a more sound approach in this regard [15]. 
There are also several potential weaknesses of the DSM-5, which have not yet been fully investigated. The primary criticism of the new diagnostic approach is related to whether or not SUDs truly are unidimensional phenomena. To demonstrate this point, MacCoun [16•] recently conducted a review of the assumptions underlying popular analyses of the criteria. At the heart of this discussion is the core supposition that a mild SUD is simply a less serious manifestation of the same syndrome as a severe SUD. If this truly is the case, and a unidimensional approach accurately captures SUDs, then all criteria are weighted equally serving as simple additive representations of a larger construct. In reality, however, there may be cardinal indicators of different levels of SUDs with respect to severity.

Preliminary evidence suggests that some criteria may be more indicative of a more severe clinical syndrome than others. In fact, a recent study demonstrated that 5 of the 11 criteria were observed almost exclusively among cases meeting criteria for a severe SUD: (1) experiencing withdrawal symptoms; (2) desire and/or inability to reduce or stop use; (3) forfeiture of recreational or other activities because of substance use; (4) failure to fulfill major role obligations at home, school, or work; and (5) craving, strong desire, or urge to use a substance [7•]. It is possible these five criteria represent an underlying 'loss of control' concept which has been generally linked to addiction [17]. The most severe SUDs are products of interactions between physiology (including, but not limited to genetic predisposition to addiction) and compulsive substance use over an extended period of time. Such factors are likely to be related to greater biological and psychological responsiveness to certain substances, along with greater levels of craving. This, in turn, would account for the inability to limit or stop use. Given the likelihood of this loss of control, it remains likely that an individual will experience several serious negative consequences of their persistent use.

These five criteria are indicative of a highly problematic pattern of substance use for many reasons. It is unlikely, for example, any one of these criteria exists in the absence of the others, especially in severe SUDs. In other words, it is nearly impossible to experience physiological withdrawal from a substance in the absence of heavy and prolonged substance use or the inability to control use without craving. A high level of use is also most likely to contribute to the relatively serious problems underlying the sacrifice of certain activities and failure to meet one's social or professional obligations. Although these preliminary findings appear to support this underlying constellation of certain criteria among severe SUDs, prospective research is clearly warranted.

On the other hand, some of the criteria are more common among the mild and severe diagnostic groups and even among those who do not receive a DSM-5 diagnosis. The development of tolerance and use in situations that are dangerous are among those criteria more likely to be present among these groups [7•]. For example, it is possible to drink heavily and not demonstrate indications of loss of control and yet develop tolerance as a result of the drinking pattern. In the case of use in situations in which it is physically hazardous (e.g., driving an automobile when impaired by substance use), we may be seeing some confounding with characteristics of risk-taking or tendency to engage in impulsive behaviors. Criteria that are more likely to exist in the absence of others, or those that are distinguished from other factors, may be more likely to be present among the lower severity levels.

\section{Implications for Treatment Under the DSM-5}

Despite limited evidence regarding the clinical implications associated with the adoption of the DSM-5 criteria, its routine use in clinical treatment settings is already underway [18]. It is important to note with caution, however, transition to the DSM-5 severity index may impact treatment planning if based on the underlying assumption that there are variations of the same condition (i.e., mild, moderate, and severe SUD). This approach theoretically corresponds to treatment provisions for the same condition according to these severity levels. In reality, empirical work may eventually determine that there are different conditions with distinct prognoses and correspondingly appropriate treatment goals [19].

Diagnosis should be used as a commonly shared classification system which is used to inform individualized, clinically driven treatment [20]. Failure to use a diagnosis as a beacon in the treatment process is likely to lead to a number of untoward treatment complications, including relapse and an uninformed, discontinuous treatment plan. Instead, SUD diagnoses should clearly inform treatment planning. The challenge with the new DSM-5 SUD criteria will be in differentiating clinically effective and individualized treatment options for mild, moderate, and severe cases. Research has not yet determined how these diagnostic classifications relate to treatment outcomes, such as relapse, so a significant amount of speculation will remain until further work is done in this area.

However, logic suggests that the different diagnoses may require different treatment approaches or goals. Those in the severe SUD diagnostic group with positive responses to most of the aforementioned criteria related to the loss of control construct may require abstinence as a treatment goal to achieve stable remission. Conversely, those with a mild diagnosis and no positive indications on the aforementioned criteria might be candidates for moderating use successfully.

It is certain, however, that some cases which would have gone previously undiagnosed under the DSM-IV will now be eligible for treatment according to the DSM-5. Diagnostic orphans, who did not previously qualify for a SUD diagnosis under the DSM-IV because they endorsed one to two DSMIV dependence criteria and did not meet the three-criterion 
threshold, will now be assigned a mild SUD diagnosis according to the DSM-5. At first glance it may seem as though clinicians have been given a wider net to cast and this may very well happen in some less serious cases. Nonetheless, research has shown that individuals who endorsed one or two alcohol dependence criteria (under the DSM-IV approach which did not yield a diagnosis and who will now be designated as a DSM-5 mild SUD) were still likely to experience adverse life events and worse physical health conditions, relative to those who went undiagnosed, which may have been ameliorated with treatment [21].

Other individuals who previously received a diagnosis based on the DSM-IV may now no longer qualify for a diagnosis. A study of first-time driving under the influence (DUI)/driving while intoxicated (DWI) offenders found that a significant portion of these cases acknowledged only driving under the influence (reflective of the DSM-IV criterion related to use in dangerous situations) and the legal problem of being arrested [22]. With the DSM-IV, these cases would have received an abuse diagnosis, but will not receive a diagnosis with the DSM-5. The same circumstances would relate to any DSM-IV abuse cases positive on only a single criterion in the absence of any dependence criteria; these will no longer receive a SUD diagnosis according to the DSM-5. This translates to a sizeable number of clients being ineligible for treatment admission due to the lack of a formal diagnosis.

This issue also may have some rather profound implications in clinical community settings, given these cases would no longer qualify for insurance reimbursement under the DSM-5. Substance use and corresponding criminal behaviors may be a major factor in a substantial number of incarcerations; however, in the absence of other positive criteria, these individuals would not meet DSM-5 diagnostic criteria. In institutional settings, such as the criminal justice system, these cases are likely to go unnoticed until their problematic substance use and related problems escalate and they subsequently experience additional social, psychological, and physical problems that substantiate a formal diagnosis.

The aforementioned criteria primarily found among severe cases should also be given special consideration with respect to potential treatment options. If favorable outcomes are to be demonstrated, treatment programs must recognize that these criteria are likely to appear in the most severe SUD cases. One viable treatment option may be to emphasize the importance of complete abstinence as an appropriate individual treatment goal given the nature and extent of the failure of control over substance use for these cases. Chronic exposure to illicit drugs and alcohol has been found to alter activity in several areas of the brain, including, most notably, the prefrontal cortex, which has been implicated in motivation, impulse control, and decision making, while fostering other associative processes, such as instrumental conditioning [17]. This is of paramount importance for severe SUD cases because instrumental conditioning toward substance use in a state of withdrawal is likely to trigger persistent use despite the occurrence of significant and pervasive adverse consequences. Thus, severe SUD cases most likely represent a more challenging population to treat, and a group in which the only way to reorient neurobiological processes away from motivation toward substance use would be the adoption of complete abstinence as a primary goal for treatment.

Cases who endorse other criteria, in comparison, may not necessarily suffer from similar neurobiological mechanisms. A hypothetical case which also contains five, albeit different, criteria may involve an individual who indicated (1) spending a great deal of time using, (2) tolerance, (3) unplanned use, (4) use in a hazardous situation, and (5) recurrent interpersonal conflicts related to use. Such a case might simply be a heavy drinker who spends a great deal of time drinking with friends, subsequently develops tolerance, occasionally drives when it is inappropriate, and has resulting conflicts with a spouse. These indicators, while precisely the same in number, are qualitatively different from cases endorsing criteria that are possibly associated with the loss of control construct. A similar level of loss of control, related to underlying neurobiological changes due to substance use, may not necessarily be exhibited. Instead, these outcomes are likely related to a trend toward substance misuse in certain circumstances, many of which involve irresponsible and impulsive behaviors (e.g., unplanned use, use in hazardous situations such as impaired driving, recurrent interpersonal conflicts related to use). The adoption of a harm reduction approach involving moderation or monitored substance use may be a more reasonable initial treatment goal for these individuals.

In sum, placing SUDs on a continuum seems to be a progressive step. Evidence demonstrating the hierarchical arrangement of abuse and dependence did not accurately depict reality. A notable case in point is the failure to fulfill role obligations, which was an abuse criterion in the DSM-IV. As noted previously, positive findings on this criterion are most often found among those positive on six or more of the eleven DSM-5 criteria.

Now there are a different set of questions which need to be answered. First, does the severity index - defined as the total number of diagnostic criteria - truly represent variations of the same disorder? Or, are we dealing with distinct disorders that manifest common symptoms and behaviors along a defined continuum? For example, during a depressive episode, individuals with either major depressive disorder or bipolar I disorder may exhibit similar symptoms. However, treatments required to achieve sustained remission for the two conditions are substantially different. Second, are there subsets of criteria which are more likely to represent certain severity levels? If so, how can these criteria best inform treatment programs to reduce relapse and achieve optimal outcomes? Third, how should severity level inform the development of individualized 
treatment goals? Is total abstinence the best practice across the board or should other options be considered for less severe cases? These are just a few of the many questions which will hopefully be answered as additional evidence surfaces regarding the implications of the DSM-5 diagnostic criteria.

\section{Conclusion}

In conclusion, while the DSM-5 may represent an advancement in the understanding and treatment of SUDs, substantial applied research will be required to determine the practical implications of the changes. Much of this can be done in routine clinical settings by more precise documentation of the positive criteria for each SUD diagnosis (i.e., mild, moderate, and severe) and monitoring treatment response in accord with initial outcomes. Sophisticated and routine treatment evaluations may be another step toward determining how well the DSM-5 informs treatment prognoses and outcomes.

\section{Compliance with Ethics Guidelines}

Conflict of Interest Albert M. Kopak, Steven L. Proctor, and Norman G. Hoffmann declare they have no conflict of interest.

Human and Animal Rights and Informed Consent This article is not based on any study of human or animal subjects performed by any of the authors.

\section{References}

Papers of particular interest, published recently, have been highlighted as:

- Of importance

1. American Psychiatric Association. Diagnostic and statistical manual of mental disorders. 5th ed. Washington, DC: American Psychiatric Association; 2013.

2. American Psychiatric Association. Diagnostic and statistical manual of mental disorders. 4th ed. Text revision. Washington, DC: American Psychiatric Association; 2000.

3. Martin CS, Steinley DL, Vergés A, Sher KJ. The proposed 2/11 symptom algorithm for DSM-5 substance-use disorders is too lenient. Psychol Med. 2011;41:2008-10.

4. Schuckit MA. Editor's corner: editorial in reply to the comments of Griffith Edwards. J Stud Alcohol Drugs. 2012;73:521-2.

5. Agrawal A, Heath AC, Lynsky MT. DSM-IV to DSM-5: the impact of proposed revisions on diagnosis of alcohol use disorders. Addiction. 2011;106:1935-43.

6. Chung T, Martin CS, Maisto SA, et al. Greater prevalence of proposed DSM-5 nicotine disorder compared to DSM-IV nicotine dependence in treated adolescents and young adults. Addiction. 2011;107:810-8.

7. Kopak AM, Metz AV, Hoffmann NG. Alcohol use disorder diagnoses in the criminal justice system: an analysis of the compatibility of current DSM-IV, proposed DSM-5.0, and DSM-5.1 diagnostic criteria in a correctional sample. Int J Offender Ther Comp Criminol. 2014;58:638-54. doi:10.1177/0306624X13485929. Kopak and colleagues examined the association between DSM-IV abuse and dependence criteria in relation to the DSM-5 alcohol use disorder severity index. The study sample included 6,871 adult male inmates and 801 adult female inmates. The most important finding was some criteria may serve as cardinal indicators of more serious alcohol-related problems compared with others.

8. Kopak AM, Proctor SL, Hoffmann NG. An assessment of the compatibility of DSM-IV and proposed DSM-5 criteria in the diagnosis of cannabis use disorders. Subst Use Misuse. 2012;47: 1328-38.

9. Mewton L, Slade T, McBride O, et al. An evaluation of the proposed DSM-5 alcohol use disorder criteria using Australian national data. Addiction. 2011;106:941-50.

10. Peer K, Rennert L, Lynch KG, et al. Prevalence of DSM-IV and DSM-5 alcohol, cocaine, opioid, and cannabis use disorders in a largely substance dependent sample. Drug Alcohol Depend. 2013;127:215-9.

11. Proctor SL, Kopak AM, Hoffmann NG. Compatibility of current DSM-IV and proposed DSM-5 diagnostic criteria for cocaine use disorders. Addict Behav. 2012;37:722-8.

12. Proctor SL, Kopak AM, Hoffmann NG. Cocaine use disorder prevalence: From current DSM-IV to proposed DSM-5 diagnostic criteria with both a two and three several level classification system. Psychol Addict Behav. Advance online publication. 2013; doi:10. 1037/a0033369 Proctor et al. examined the relationship between DSM-IV abuse and dependence criteria relative to the DSM-5 substance use disorder severity index. The study was based on observations with 6,871 adult male inmates. One important finding was nearly all (91\%) of DSM-IV dependence cases met DSM-5 criteria for severe cocaine use disorders. The other important finding was DSM-IV abuse cases who also endorsed one or two dependence criteria were largely divided between mild and moderate substance use disorders under DSM-5 criteria.

13. Muthén B. Should substance use disorders be considered as categorical or dimensional? Addiction. 2006;101:6-16.

14. Hasin DS. Combining abuse and dependence in DSM-5. J Stud Alcohol Drugs. 2012;73:702-4.

15. Hasin DS, O'Brien CP, Auriacombe M, et al. DSM-5 criteria for substance use disorders: recommendations and rationale. Am J Psychiatr. 2013;170:834-51.

16. MacCoun RJ. The puzzling unidimensionality of DSM-5 substance use disorder diagnoses. Front Psychiatry. 2013;4:153. MacCoun addressed the competing models used to assess the related, yet distinct criteria in substance use disorder diagnoses. Existing research was grouped and discussed according to whether a Reflective model, Formative model, Guttman scale, Causal chain, Causal model, or Network model offered superior fit and accurate representation of the dimensionality of the DSM-5 approach. MacCoun concluded these models should be used to ultimately reach a deeper understanding of the true nature of the covariance of substance use disorder diagnostic criteria.

17. Belin D, Belin-Rauscent A, Murray JE, Everitt BJ. Addiction: failure of control over maladaptive incentive habits. Curr Opin Neurobiol. 2013;23:564-72.

18. Mee-Lee D, editor. ASAM criteria: treatment criteria for addictive, substance-related, and co-occurring conditions. Carson City: The Change Companies; 2013.

19. Hoffmann NG, Hoffmann TD. Construct validity for alcohol dependence as indicated by the SUDDS-IV. J Subst Use Misuse. 2003;38:293-306.

20. Mee-Lee D. What's in it for me? The new ASAM criteria and how DSM-5 fits. Paper presented at ODAPCA's 38th Fall Conference, 
Norman, OK. 2013; retrieved from http://corpsok.com/odap/sites/ default/files/HO.1.5h.WhatsInForMe.TheNewASAM\%20Criteria. HowDSM5Fits.Norman.OK_.10.3.13.pdf

21. McBride O, Adamson G, Bunting B, McCann S. Diagnostic orphans: comparing self-report lifetime course to groups with
DSM-IV alcohol abuse and dependence. Addict Behav. 2009;34:86-91.

22. Baley JW, Hoffmann NG. The impact of the proposed changes for the DSM-5 on diagnoses of DUI/DWI offenders. 2014; Manuscript submitted for publication. 